\title{
Thickness-Tunable Self-Assembled Colloidal Nanoplatelet Films Enable Ultrathin Optical Gain Media
}

\author{
Onur Erdem, Sina Foroutan, Negar Gheshlaghi, Burak Guzelturk, Yemliha Altintas, \\ and Hilmi Volkan Demir*
}

Cite This: Nano Lett. 2020, 20, 6459-6465

Read Online

ABSTRACT: We propose and demonstrate construction of highly uniform, multilayered superstructures of $\mathrm{CdSe} / \mathrm{CdZnS}$ core/shell colloidal nanoplatelets (NPLs) using liquid interface self-assembly. These NPLs are sequentially deposited onto a solid substrate into slabs having monolayer-precise thickness across tens of $\mathrm{cm}^{2}$ areas. Because of near-unity surface coverage and excellent uniformity, amplified spontaneous emission (ASE) is observed from an uncharacteristically thin film having 6 NPL layers, corresponding to a mere $42 \mathrm{~nm}$ thickness. Furthermore, systematic studies on optical gain of these NPL superstructures having thicknesses ranging from 6 to 15 layers revealed the gradual reduction in gain

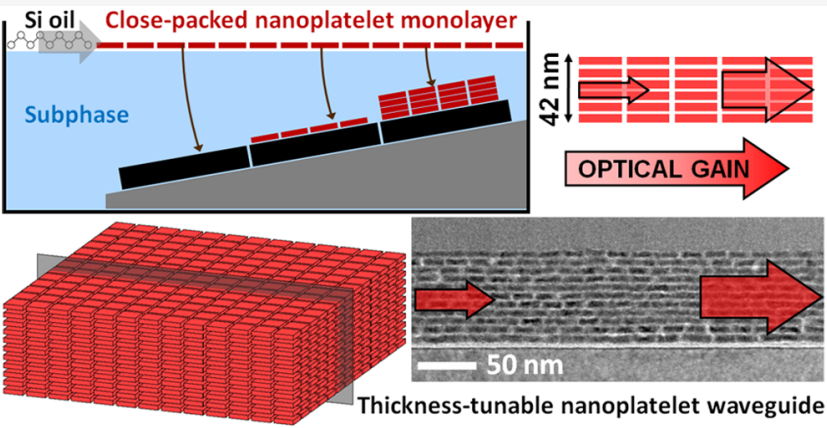
threshold with increasing number of layers, along with a continuous spectral shift of the ASE peak $(\sim 18 \mathrm{~nm})$. These observations can be explained by the change in the optical mode confinement factor with the NPL waveguide thickness and propagation wavelength. This bottom-up construction technique for thickness-tunable, three-dimensional NPL superstructures can be used for large-area device fabrication.

KEYWORDS: liquid interface self-assembly, colloidal nanoplatelets, planar waveguides, optical gain, amplified spontaneous emission

$\mathrm{O}$ ptical gain in colloidal semiconductor nanocrystals (NCs) has thus far been thoroughly investigated in terms of their gain performance and potential use as lasing media. ${ }^{1-5}$ Colloidal quantum wells, also known as nanoplatelets (NPLs), are the most recent class among these colloidal semiconductor NCs, which offer favorable properties for lasing, including narrow emission line width due to their atomically flat lateral surfaces and enhanced oscillator strength. ${ }^{6}$ Soon after their introduction, NPLs have been shown to be an excellent family of nanoemitters for lasing as they feature suppressed inhomogeneous broadening, ${ }^{6,7}$ reduced gain thresholds ${ }^{4,8,9}$ and escalated gain coefficients. ${ }^{10}$ Since then, NPLs have been extensively studied for optical gain and lasing. $4,11-14$

Solid films of nanoemitters require a minimum thickness to display optical gain, which is typically achieved via spin-coating or drop-casting of a concentrated NC solution onto the substrate. However, drop-casting technique commonly leads to nonuniform films with possibly large variance in thickness across large area films. Although spin-coating generally produces uniform films with a well-defined thickness, controlling the film thickness via experimental parameters such as rotation speed of the spinner and amount and concentration of NCs is challenging in terms of precision and reproducibility, particularly in the case of anisotropic NCs (i.e., nanorods and NPLs) that can exhibit distinct in-film orientations. ${ }^{15,16}$ Liquid-air interface selfassembly was utilized to control the orientation of the NPLs and deposit NPL films with uniform orientation (i.e., either all face-down or all edge-up); ${ }^{17-20}$ however, these earlier efforts either focused on deposition of single layered films or lacked exact control over film thickness. As a result, creating thicknesstunable NPL films and studying and understanding their optical gain by taking their thickness into account is cumbersome.

To address these issues, we propose and demonstrate the construction of superstructures of NPLs, all oriented face-down with a precise number of complete NPL monolayers using liquid-air interface self-assembly to create large-scale (several tens of $\mathrm{cm}^{2}$ ) and thickness-controlled (1- to 15-monolayered) NPL films. To this end, densely packed monolayers of NPLs are deposited on substrates repetitively via this self-assembly deposition, yielding excellent homogeneity throughout the film. This sequential deposition of NPLs enables construction of large-area slabs of NPLs with precisely controlled thickness, which can be utilized as optically active structures.

Equipped with the ability to construct thickness-controlled NPL superstructures, we systematically studied their optical gain

Received: May 20, 2020

Revised: July 31,2020

Published: July 31, 2020 
properties as a function of the number of NPL layers. The dense packing and high uniformity of our deposited NPL superstructures on fused silica enable the observation of optical gain with only six NPL layers, which have a thickness of $42 \mathrm{~nm}$. This film thickness is much smaller than the previously reported thicknesses typically of a few hundreds of nanometers for the NC films displaying optical gain ${ }^{3,10,21,22}$ and, to the best of our knowledge, the thinnest ever reported optical gain medium without using any optical feedback on a bare substrate. We find that the observation of optical gain is limited to a critical thickness, below which no waveguiding mode within the NPL slab exists. By increasing the number of NPL layers in the film further beyond the critical thickness, we were able to observe a gradual decrease in the gain threshold, which can be explained by progressively increasing field confinement factor within the NPL slab. Our results show that NPLs as well as other types of NCs can benefit from this sequential liquid-air interface deposition technique for the creation of two- or threedimensional (3D) pure or hybrid NC superstructures.

\section{RESULTS AND DISCUSSION}

CdSe NPLs of 4.5 monolayer atomic thickness were synthesized according to the previous literature. ${ }^{6,7}$ These cores were used in the synthesis of $\mathrm{CdSe} / \mathrm{Cd}_{0.25} \mathrm{Zn}_{0.75} \mathrm{~S}$ core/alloyed-shell NPLs via hot-injection technique, as previously reported by our group (see Supporting Information). ${ }^{9,23}$ The transmission electron microscopy (TEM) image in Figure la shows that the NPLs
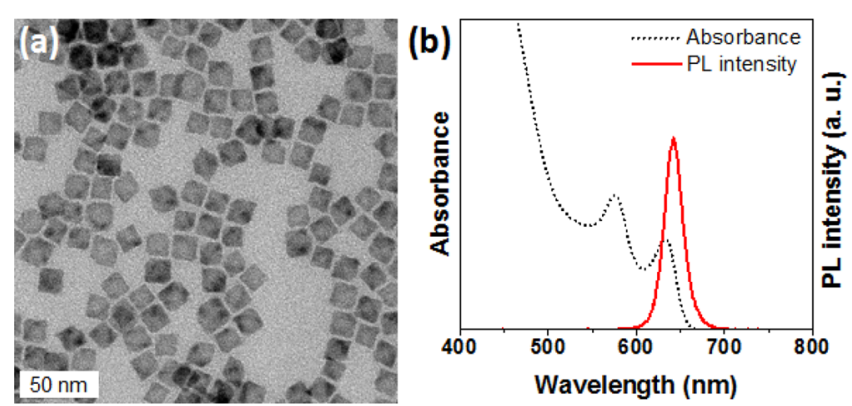

Figure 1. (a) Transmission electron micrograph of square-shaped $\mathrm{CdSe} / \mathrm{Cd}_{0.25} \mathrm{Zn}_{0.75} \mathrm{~S}$ core/shell NPLs. (b) Absorbance and photoluminescence spectra for the $\mathrm{CdSe} / \mathrm{Cd}_{0.25} \mathrm{Zn}_{0.75} \mathrm{~S}$ NPLs.

have squarelike lateral shape with a side length of $17.8 \pm 1.5 \mathrm{~nm}$ and a thickness of $\sim 4.1 \mathrm{~nm}$ as determined from TEM imaging (see Figure S1). The absorbance and photoluminescence (PL) spectra of these NPLs are presented in Figure $1 \mathrm{~b}$. The first and second excitonic peaks reside at 631 and $573 \mathrm{~nm}$, respectively, whereas the PL peak is at $642 \mathrm{~nm}$.

To deposit these NPLs into thickness-controlled thin films, we modified our previously reported self-assembly procedure ${ }^{19}$ and applied it sequentially as many times as needed to reach the desired number of NPL layers in the construction. The process is schematically illustrated in Figure 2a. Starting with precleaned blank substrates treated with $1 \mathrm{H}, 1 \mathrm{H}, 2 \mathrm{H}, 2 \mathrm{H}$-perfluorodecyltriethoxysilane $^{24}$ (see Supporting Information) or continuing with previously deposited substrates attached to a glass stage tilted by about $10^{\circ}$, we fully immerse the stage and the substrates into the subphase of diethylene glycol (DEG). Twenty-five microliters of hexane solution of NPLs with a concentration of $\sim 5.5 \times 10^{-7} \mathrm{M}$ is then dropped onto DEG, resulting in the liquid interface being almost fully covered with a membrane of NPLs after hexane is fully evaporated. To create and maintain a compact membrane, we used silicone oil as a suspension material to create surface pressure, which helps compress the deposited NCs, yielding a close-packed NPL film, as was previously used for preparing a close-packed film of gold nanoparticles. ${ }^{25}$ In our case, we dropped the silicone oil (silicone elastomer, Sylgard 184) dissolved in hexane from one edge to compress the membrane (see SI Video). The subphase is then slowly drained with the help of a peristaltic pump at a rate of $50 \mu \mathrm{L} / \mathrm{min}$, corresponding to $\sim 260 \mathrm{~nm}$ descent of liquid level per second in the Teflon dish with $64 \mathrm{~mm}$ diameter used for the deposition. As the subphase is taken out, the membrane of NPLs eventually sinks onto the substrates while DEG is drawn away from the substrates. The surface treatment, which renders the substrates hydrophobic, as well as their tilted placement help facilitate the repulsion of the subphase during the transfer of the NPL membrane onto the substrate. After the draining is complete, the residual DEG, if any, on the substrates is evaporated under vacuum at room temperature.

At the end of this procedure, one full monolayer of NPLs is deposited onto multiple substrates, as seen in the scanning electron microscopy (SEM) image of one of the substrates shown in Figure 2b. The NPLs form a compact monolayer with excellent uniformity with no visible multilayers or aggregation. Furthermore, because of the compression by silicone oil the deposited NPLs are densely packed, yielding near-unity surface coverage. Without the assistance of silicone oil, only a fraction of the NPLs on the membrane sticks to the surface during the transfer process, yielding submonolayer coverage (see Figure S2). To further demonstrate the applicability of this technique to cover arbitrarily large areas, we deposited a single monolayer of NPLs onto a 4 in. fused silica wafer. The photograph in Figure $2 c$ displays the luminescence across the wafer under UV excitation, where an area of about $80 \mathrm{~cm}^{2}$ is uniformly deposited with a monolayer of NPLs.

In order to create thicker films with multiple NPL layers, this self-assembly procedure is repeated on the same previously deposited substrates as many times as desired. The resulting films maintain their strong emission and high uniformity. A cross-sectional TEM image taken from an exemplary case of 11 NPL layers, seen in Figure 2d, reveals that the NPL layers remain intact and do not introduce roughness as they build up. All of the NPL layers are distinctly visible, separated by their surface ligands. The uniformity of these layers continues to persist even over larger areas (see Figures S3 and S6). Atomic force microscopy (AFM) imaging shows that the roughness of these multilayer films is limited to at most a few nanometers even for the thickest NPL films (Figure S4b).

Ellipsometric measurements on the thicknesses of multilayered NPL films having different numbers of layers verify the linear trend between the film thickness and the number of NPL layers (Figure S4a). We extracted from the slope of the linear fit that each deposited monolayer adds up $7.0 \mathrm{~nm}$ to the film thickness. Because the thickness of the core/shell NPL structure is $\sim 4.1 \mathrm{~nm}$, the ligand brush between the NPL layers is deduced to be $\sim 2.9 \mathrm{~nm}$ thick, which indicates a minor interdigitation between the ligand brushes of oleic acid/oleylamine. This interdigitation would allow the NPLs to stick together via van der Waals interaction without having to deposit an oppositely charged linker molecule between the monolayers, as is commonly employed for layer-by-layer NC deposition. ${ }^{26-29}$ Not having to rely on the deposition of oppositely charged particles adds up to the versatility of our multilayer deposition technique as the thickness of such oppositely charged bilayers 


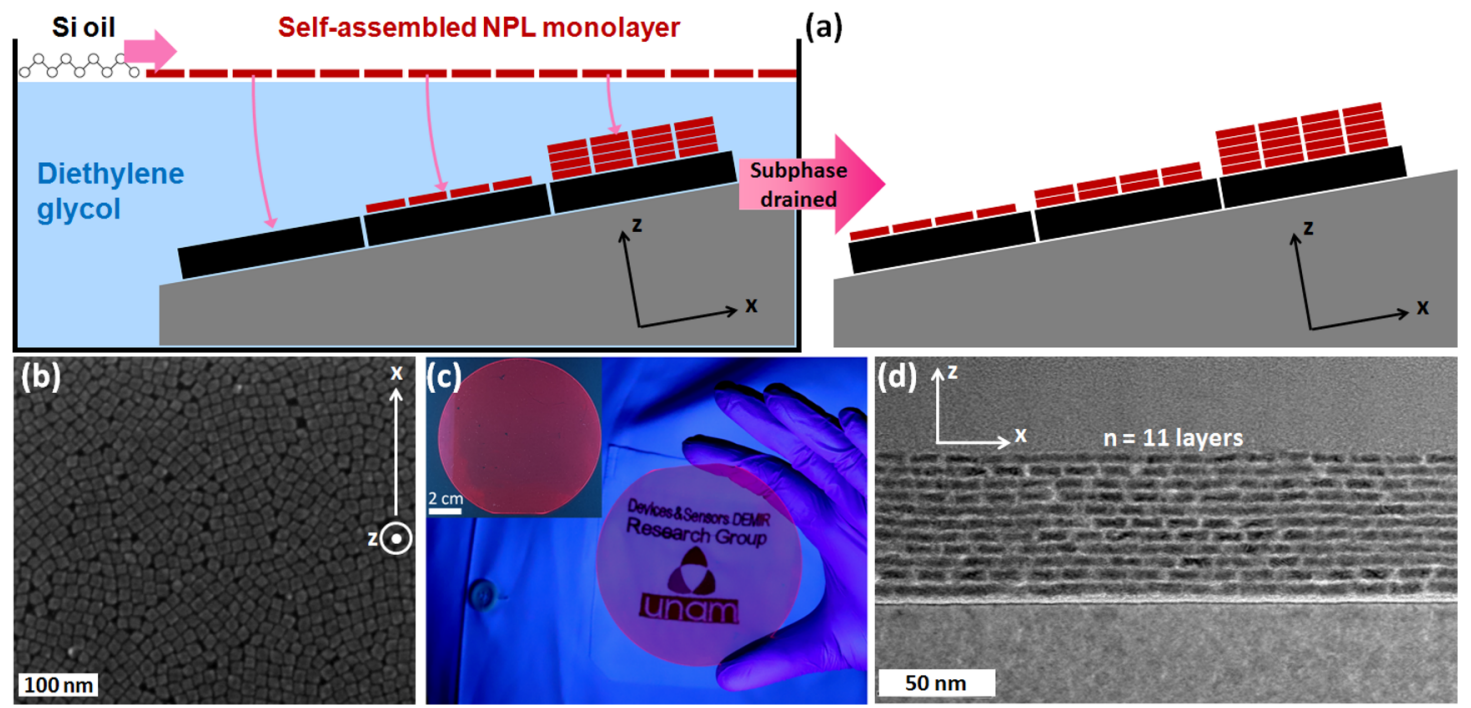

Figure 2. (a) Schematic demonstration of liquid-air interface self-assembly of the NPLs. Blank or predeposited substrates are inserted into a subphase of diethylene glycol. The NPL solution is dropped onto the subphase and quickly spreads across the liquid-air interface. Dropping silicone oil causes compression of the NPL membrane. After the subphase is drained, all of the substrates are deposited with one additional layer of NPLs. (b) Scanning electron micrograph of one monolayer of NPLs deposited through liquid-air interface self-assembly. (c) Photograph of one monolayer of NPLs deposited onto 4 in. fused silica illuminated under UV light. Logos of Demir Group and UNAM used with permission. (d) Cross-sectional TEM image of the 11 NPL monolayers sequentially deposited onto silicon. All the NPL layers are distinctly visible, separated by their surface ligands.

can be altered by the ambient conditions, ${ }^{30}$ or by unintentional diffusion of charged particles into the previously deposited inner layers during dipping steps, which leads to superlinear thickness growth. $^{31}$ Our self-assembly technique thus presents an alternative, robust approach to the existing methods ${ }^{17,32-34}$ of $\mathrm{NC}$ self-assembly at liquid interface while maintaining large-area uniformity and precision in film thickness.

To characterize these NPL slabs in terms of their optical gain performance, we deposited NPL multilayers through the aforementioned procedure onto fused silica substrates with a varying number $(n)$ of NPL layers up to $n=15$. We were able to observe amplified spontaneous emission (ASE) from these films with $n=6$ and above, whereas no ASE has been observed for $n \leq$ 5. We therefore present our results for the films ranging from $n=$ 6 (42 nm thick) to $n=15$ (105 nm thick). We use a stripe excitation configuration for the ASE measurements, where the optical pump is normally incident on the specimen and the PL emission is collected from the side (see Supporting Information). ${ }^{35}$ Our incident pump has $400 \mathrm{~nm}$ wavelength with $1 \mathrm{kHz}$ pulse rate and $\sim 110 \mathrm{fs}$ pulse width. The PL spectra were collected at various pump fluences for each $n$. These spectra are shown in Figure 3a for $n=6,9$, and 15. ASE is evident in all three cases from the emergence of the second emission feature beyond a pumping threshold, as well as the superlinear increase of the integrated emission intensity (Figure $3 b$ ). The per pulse gain thresholds for these three cases have been determined to be 31.3, 19.3, and $7.5 \mu \mathrm{J} \mathrm{cm}^{-2}$ for $n=6,9$ and 15 , respectively. The tendency of the gain threshold to drop with increasing $n$ is also seen in Figure 3c, where the thresholds for the films of all the thicknesses from $n=6$ to $n=15$ are plotted. The gradual drop of the gain threshold continues up to $n=15$ for which it is $7.5 \mu \mathrm{J}$ $\mathrm{cm}^{-2}$. This value is also on par with the reported gain thresholds of CdSe-based core/shell NPLs synthesized with hot-injection technique. ${ }^{9,36-38}$

Another notable observation is the evolution of the spectral position of the ASE peak with respect to the spontaneous emission for varied $n$. In Figure 3d, one PL spectrum is plotted for each $n$ at pump intensities that are slightly above the respective gain thresholds. For the thinnest slabs, the ASE peak displays little or no shift with respect to the spontaneous emission peak. As $n$ keeps increasing beyond 9, however, a gradual increase in the red shift of this peak is observed until $n=$ 15 (see Figure 3d), where the ASE peak saturates at around 664 $\mathrm{nm}$ (see Figure $3 \mathrm{~d}$ and Table S1) and its red shift is determined to be $18.2 \mathrm{~nm}$. A similar change in the shift of the ASE peak with film thickness has previously been observed with the thin films composed of other types of emitters and was attributed to the change in the critical wavelength of propagation in the planar waveguides as the film thickness is varied. ${ }^{39,40}$ In our case, this shift of the ASE peak allows for fine-tuning of the optical gain wavelength across a $17 \mathrm{~nm}$ wide spectral band (see Table S1) by precisely adjusting the NPL waveguide thickness.

Finally, we demonstrate polarization in the ASE of ultrathin NPL films. As two exemplary cases, the emission intensity versus the polarizer angle has been plotted for 11- and 15-layered NPL films in Figure $3 \mathrm{e}$. The emission from the 11-layered film is observed to be highly polarized with a polarization of 0.97 , whereas the polarization of the ASE reduces to 0.60 for the 15layered film. The evolution of the ASE polarization with film thickness is plotted in Figure $3 \mathrm{f}$, where we see a significant decrease after $n=13$, which is attributed to the emergence of the TM mode in the thickest NPL films (i.e., $n \geq 13$ ) as further discussed below.

To elaborate on the observed characteristics of the NPL slabs, we employed a numerical analysis of guided modes within the NPL layer (see Supporting Information). Approximating the NPL multilayer as a homogeneous medium, our structure can be modeled as an asymmetric planar waveguide confined between semi-infinite fused silica and air (Figure 4a). As the refractive index of the NPL layer, which is measured as $n_{2}=1.96$ via ellipsometry, is greater than those of air $\left(n_{1}=1\right)$ and fused silica $\left(n_{3}=1.45\right)$, this configuration will allow propagation of the supported modes through the NPL layer via total internal reflection. However, the existence of these modes requires a 

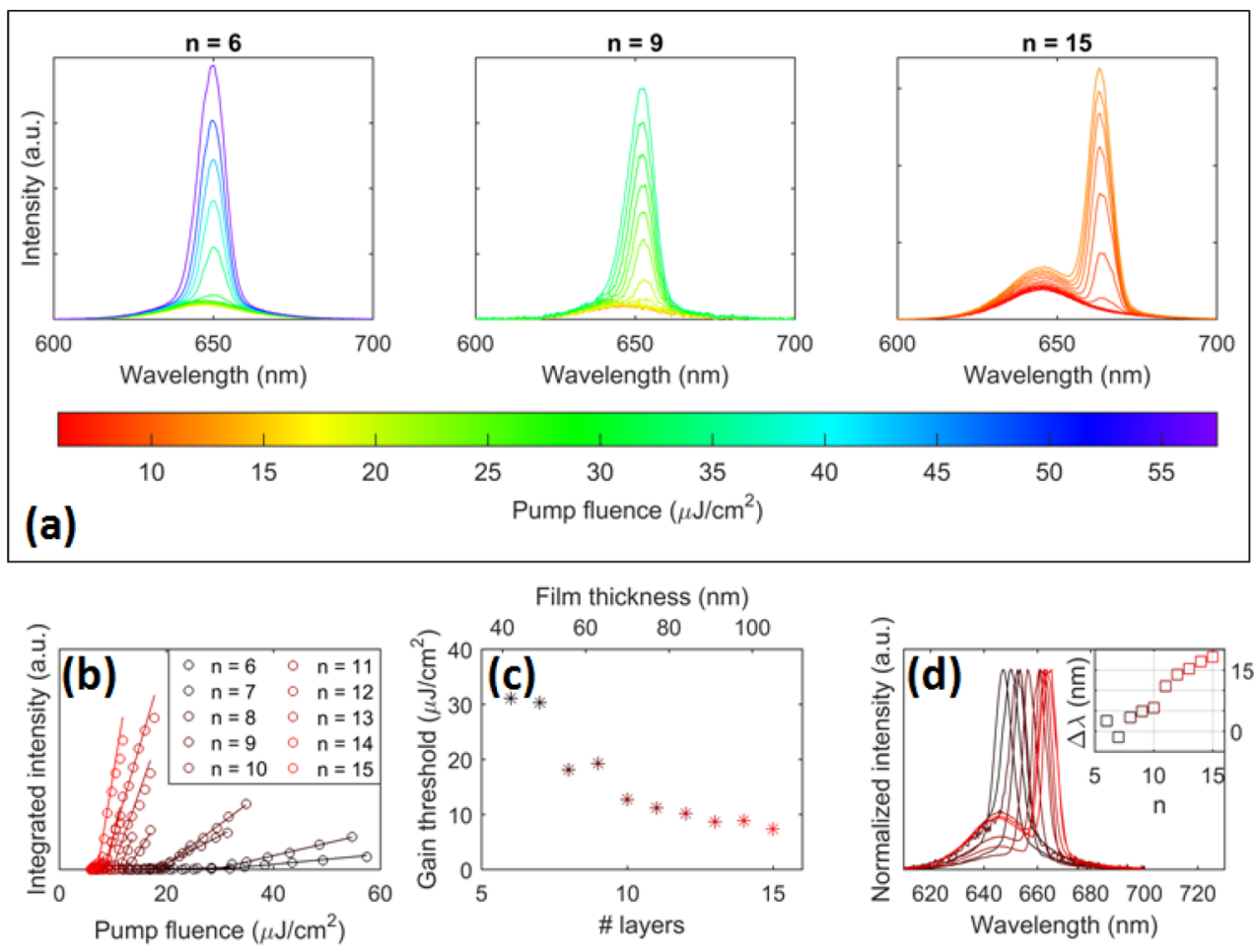

(e)

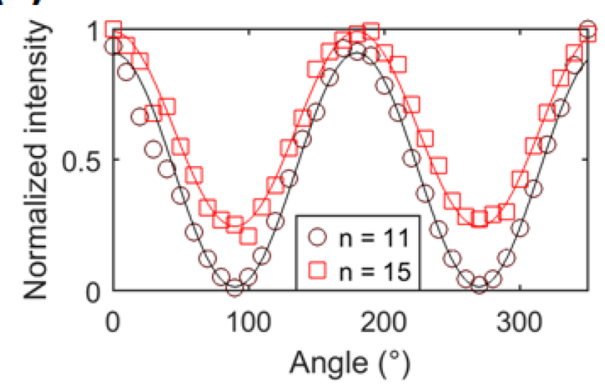

(f)

Thickness $(\mathrm{nm})$

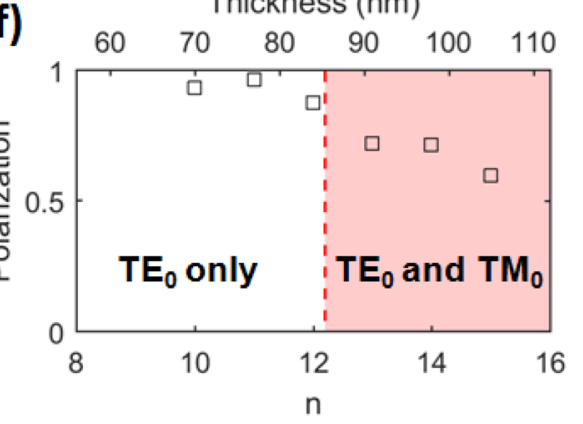

Figure 3. (a) Emission spectra of the NPL films having a different number $n$ of layers excited with a pulsed laser at $400 \mathrm{~nm}$ : left, $n=6$; center, $n=9$; right, $n=15$. Colorbar at the bottom is common for all three plots. (b) Integrated intensity as a function of the pump fluence for all the NPL films from $n=6$ to $n=15$. (c) Evolution of the optical gain threshold with the number of layers $n$ as deduced from the data in panel b. (d) Shifting of the ASE peak with respect to the spontaneous emission for different $n$. Inset shows the difference between ASE and spontaneous emission peaks. The color coding is identical in panels $\mathrm{b}-\mathrm{d}$. (e) Polarization measurements for the ASE from 11- (black) and 15- (red) layered NPL films. (f) Degree of polarization of ASE in NPL superstructures as a function of the number of NPL layers $n$. Dashed line indicates the critical thickness for $\mathrm{TM}_{0}$ mode $(85.4 \mathrm{~nm})$.

minimum thickness, which can be calculated using the wellestablished planar waveguide formalism. Accordingly, the critical thickness $t_{c}$ of the waveguide necessary for the presence of the $m$ th order transverse electric and transverse magnetic field modes $\left(\mathrm{TE}_{\mathrm{m}}\right.$ and $\left.\mathrm{TM}_{\mathrm{m}}\right)$ is given by

$$
\begin{aligned}
& t_{\mathrm{c}, \mathrm{TE}}=\frac{\lambda}{2 \pi \sqrt{n_{2}^{2}-n_{3}^{2}}}\left[m \pi+\tan ^{-1}\left(\sqrt{\frac{n_{3}^{2}-n_{1}^{2}}{n_{2}^{2}-n_{3}^{2}}}\right)\right] \\
& t_{\mathrm{c}, \mathrm{TM}}=\frac{\lambda}{2 \pi \sqrt{n_{2}^{2}-n_{3}^{2}}}\left[m \pi+\tan ^{-1}\left(\frac{n_{2}^{2}}{n_{1}^{2}} \sqrt{\frac{n_{3}^{2}-n_{1}^{2}}{n_{2}^{2}-n_{3}^{2}}}\right)\right]
\end{aligned}
$$

where $\lambda$ is the wavelength of the mode in free space. ${ }^{41}$ For $\lambda=$ $650 \mathrm{~nm}$, eq 1 reveals that the minimum thickness required for the $\mathrm{TE}_{0}$ mode is $52.7 \mathrm{~nm}$, which is in close proximity to the experimental onset of ASE in our multilayered NPLs observed with six layers (thickness of $42 \mathrm{~nm}$ ). The slight discrepancy between the experimental critical thickness and the calculated minimum thickness at which ASE is observed could be due to the effective refractive index of the NPL medium being modified under hard pumping conditions. The fact that optical gain is observed at $42 \mathrm{~nm}$ shows that the actual refractive index of the NPL film is greater than 1.96 and nonzero modal confinement is possible at this thickness. For numerical convenience, we therefore take $n_{2}=2.10$ for mode calculations that will follow. This refractive index is not far off from the one measured by ellipsometry $\left(n_{2}=1.96\right)$ and numerically yields $t_{c}=41.2 \mathrm{~nm}$ $(85.4 \mathrm{~nm})$ for $\mathrm{TE}_{0}\left(\mathrm{TM}_{0}\right)$ mode.

The calculated mode intensity profile of $\mathrm{TE}_{0}$ mode is plotted in Figure 4a for a six-layered NPL waveguide. Because of the ultrathin NPL film, only a small portion of the propagating field is confined within the gain medium. The field confinement factor, $\Gamma$, is calculated as $6.3 \times 10^{-3}$ for $n=6$. This factor monotonously increases with additional NPL layers deposited, as seen in Figure $4 \mathrm{~b}$. More notably, it undergoes a significant jump of 4 orders of magnitude from $n=5$ to $n=6$, which is in accordance with the onset of optical gain. By increasing the 

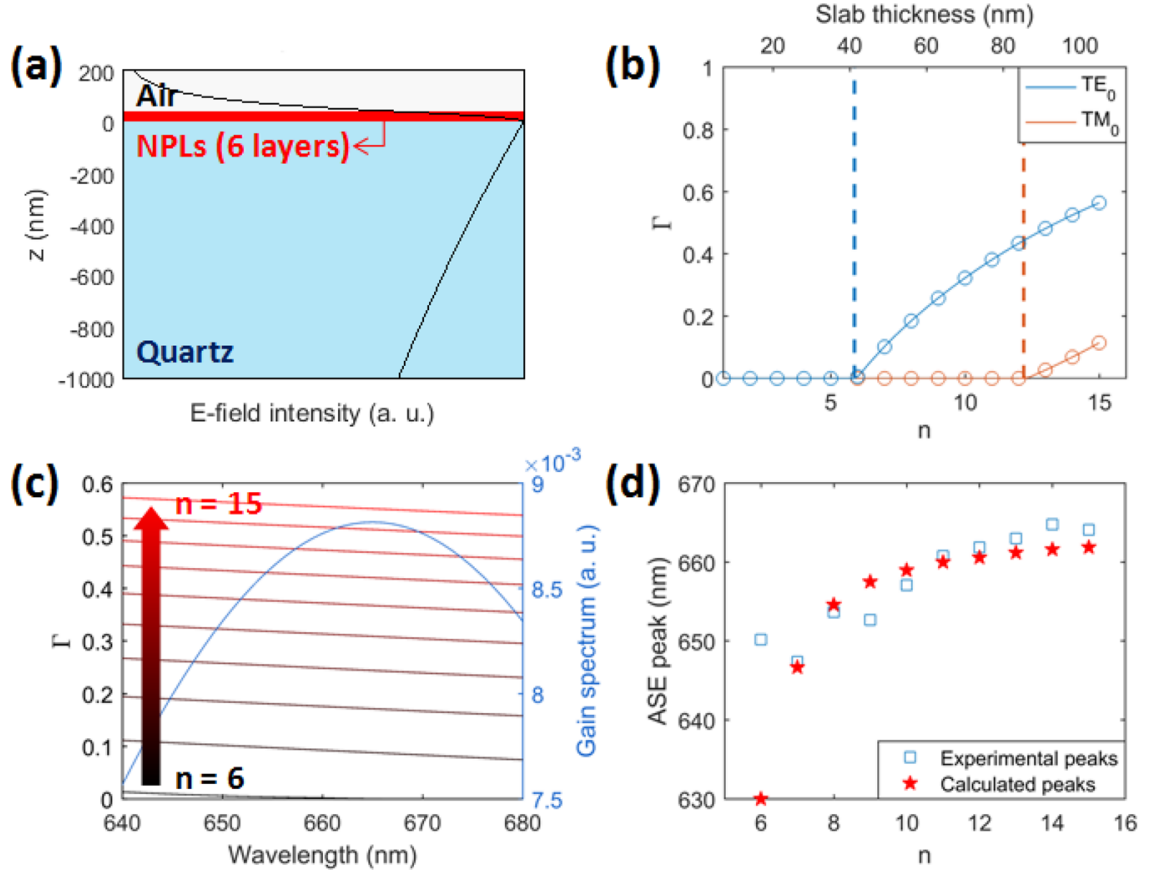

Figure 4. (a) Electric field profile of the fundamental TE mode numerically calculated for the NPL slab with $n=6$. (b) Optical confinement factor $\Gamma$ calculated at $650 \mathrm{~nm}$ for a different number of NPL layers (and slab thickness). Dashed line indicates the critical thickness of $41.2 \mathrm{~nm}$ for the existence of propagating modes within the slab. (c) Left axis: Variation of the confinement factor $\Gamma$ with wavelength for different numbers of layers. Right axis: Material gain spectrum $G(\lambda)$ for our NPLs estimated using the measured ASE peaks at different film thicknesses, modeled as a Gaussian centered at $665 \mathrm{~nm}$ with a fwhm of $106.6 \mathrm{~nm}$ (blue). (d) ASE peaks calculated as the maximum of $\Gamma(\lambda) \times G(\lambda)$ for each $n$ (red stars), together with the experimentally measured ASE peaks (blue squares).

thickness of the gain medium even further, the optical gain is facilitated by the stronger confinement of the optical mode into the NPL slab, hence the reduction in the gain threshold.

Above the critical thickness for $\mathrm{TM}_{0}$ mode, we also see the confinement of the $\mathrm{TM}_{0}$ mode starting to become significant, albeit to a lesser extent. The emergence of the $\mathrm{TM}_{0}$ mode at $n=$ 13 is consistent with the reduction in the ASE polarization at $n=$ 13 and above as seen in Figure 3f. With the $\mathrm{TM}_{0}$ mode present, part of the emission is coupled into this mode, reducing the overall polarization of the ASE. For $n<13$, however, only the $\mathrm{TE}_{0}$ mode is present, which results in highly polarized ASE.

To account for the spectral shifting of the ASE peak with film thickness, we consider the variance of the mode confinement with propagation wavelength. Previously, observing a similar trend in the ASE peak of optically pumped polystyrene films, Calzado et al. argued qualitatively that each vibronic mode experiences a different loss at different film thicknesses. ${ }^{40}$ As a result, the propagation losses for the modes having longer wavelengths are enhanced compared to those supported at shorter wavelengths, which explains the blue-shift of the ASE peak at thinner polystyrene films. ${ }^{40}$ A similar argument can be made for our NPL constructs as well, since the mode confinement near the critical thickness is highly sensitive to the wavelength, as seen in Figure 4c. Furthermore, the gain band of the NCs, which determines the spectral range at which gain can be observed from the material, is not flat in the generic case. That is, the material gain coefficient $G$ is a function of $\lambda$. The film gain coefficient will then be given by $g(\lambda)=G(\lambda) \Gamma(\lambda)$, neglecting the losses. Therefore, the ASE peak at a particular thickness can be estimated by calculating the maximum of $G(\lambda) \Gamma(\lambda)$.
To test if this simple formalism can explain the observed ASE shift, we model the gain spectrum of the material as a generalized Gaussian function

$$
G(\lambda)=G_{c} e^{-\left(\frac{\left|\lambda-\lambda_{c}\right|}{\alpha}\right)^{\beta}}
$$

where $G_{\mathrm{c}}$ is the gain coefficient at the peak wavelength $\lambda_{\mathrm{c}}$ and $\beta$ and $\alpha$ are parameters related to the shape and width of the distribution, respectively. $\lambda_{\mathrm{c}}$ is fixed at $665 \mathrm{~nm}$ since it is the peak of the most red-shifted ASE feature among all the tested multilayered films. The parameters $\beta$ and $\alpha$ are swept to find the parameter couple that minimizes the difference between the experimentally determined ASE peaks and the calculated ones. The optimum parameters found this way are $\beta=2.0$ and $\alpha=64$ for which eq 2 is plotted in Figure 4c. The calculated ASE peaks are plotted in Figure $4 \mathrm{~d}$, along with the experimentally determined peaks. We see that apart from the ASE peak for $n$ $=6$, the assumed $G(\lambda)$ can estimate the trend in the ASE peak shift fairly well. The discrepancy at $n=6$ is most likely due to the actual $G(\lambda)$ having a finite range that does not include $630 \mathrm{~nm}$. It is also worth noting that because for very thick films $\Gamma(\lambda) \approx 1$ throughout the gain band, this model can readily predict that with increasing thickness the ASE peak will converge to $\lambda_{\text {c }}$. Indeed, the experimental ASE peak seems to be saturating toward a definite red shift around $\lambda=665 \mathrm{~nm}$. Although this type of red shift is expected for NCs having type-I band alignment, ${ }^{42}$ here we show that it is possible to obtain nonshifting ASE from type-I NCs by using their ultrathin films.

\section{CONCLUSION}

We have created planar waveguides out of multilayered NPLs laid in face-down orientation by a sequentially repeated liquid interface self-assembly, which is applicable to large scales. The 
NPL films prepared with this technique have close packing and excellent uniformity over many tens of $\mathrm{cm}^{2}$ large areas and can work as optical slab waveguides of which optical confinement is revealed as a function of the number of NPL layers, or equivalently, the film thickness at monolayer precision. Our findings show that these self-assembled NPL slabs can also be used as optically active planar waveguides with precise thicknesscontrolled gain, which can find applications in on-chip active photonic devices requiring in-plane waveguided light.

In the case of electrically driven NC films, the organic surfactants in NC films act as insulating barriers that impede charge or heat transfer, limiting their use in electroluminescent devices. The thickness of our NPL films inducing optical gain is as small as $\sim 40 \mathrm{~nm}$, with only six NPL layers, may help significantly to reduce the organic barriers that should be overcome by the electric current in the vertical direction. This work may therefore serve as a foundation for developing the optically active media of electrically driven NPL lasers without having to switch to inorganic ligands. The technique presented in this work can be readily extended to other types of NCs to create large-area hybrid NC superstructures to be used for device fabrication. This work therefore paves the way for exploration of a rich variety of large-area bottom-up NC superstructures.

\section{ASSOCIATED CONTENT}

\section{(s) Supporting Information}

The Supporting Information is available free of charge at https://pubs.acs.org/doi/10.1021/acs.nanolett.0c02153.

Synthesis of CdSe/CdZnS shell NPLs, details of the optical gain experiments and mode profile calculations, description of the silane treatment for the substrates, additional SEM images for a monolayer of NPLs, additional cross sectional TEM image for NPL multilayers, thickness and roughness measurements on NPL multilayers, characterization of large-area uniformity of the samples (PDF)

Demonstration of the compression of a NPL monolayer on the liquid interface by the addition of silicone oil (AVI)

\section{AUTHOR INFORMATION}

\section{Corresponding Author}

Hilmi Volkan Demir - Department of Electrical and Electronics Engineering, Department of Physics, UNAM - Institute of Materials Science and Nanotechnology, Bilkent University, Ankara 06800, Turkey; LUMINOUS! Centre of Excellence for Semiconductor Lighting and Displays, The Photonics Institute, School of Electrical and Electronic Engineering, School of Physical and Mathematical Sciences, School of Materials Science and Engineering, Nanyang Technological University, Singapore 639798 Singapore; (1) orcid.org/0000-0003-1793-112X; Email: hvdemir@ntu.edu.sg

\section{Authors}

Onur Erdem - Department of Electrical and Electronics Engineering, Department of Physics, UNAM - Institute of Materials Science and Nanotechnology, Bilkent University, Ankara 06800, Turkey

Sina Foroutan - Department of Electrical and Electronics Engineering, Department of Physics, UNAM - Institute of Materials Science and Nanotechnology, Bilkent University, Ankara 06800, Turkey; ○ orcid.org/0000-0003-0623-8987
Negar Gheshlaghi - Department of Electrical and Electronics Engineering, Department of Physics, UNAM - Institute of Materials Science and Nanotechnology, Bilkent University, Ankara 06800, Turkey

Burak Guzelturk - Advanced Photon Source, Argonne National Laboratory, Lemont, Illinois 60439, United States; ํ orcid.org/ 0000-0003-1977-6485

Yemliha Altintas - Department of Electrical and Electronics Engineering, Department of Physics, UNAM - Institute of Materials Science and Nanotechnology, Bilkent University, Ankara 06800, Turkey; Department of Materials Science and Nanotechnology, Abdullah Gul University, Kayseri 38080, Turkey

Complete contact information is available at:

https://pubs.acs.org/10.1021/acs.nanolett.0c02153

\section{Notes}

The authors declare no competing financial interest.

\section{ACKNOWLEDGMENTS}

The authors acknowledge the financial support from the Singapore National Research Foundation under the program NRF-NRFI2016-08 and in part from TUBITAK 115E679. The authors thank Mr. Mustafa Guler for TEM imaging of the assynthesized NPLs and preparation of the TEM cross-sectional sample, Mr. Semih Bozkurt for his support on the AFM characterization, Dr. Gokce Celik for her help on the ellipsometric analyses and confocal microscopy imaging, Mr. Emre Unal for his assistance in photography of the large-area sample, Mr. Mete Duman for his assistance on the recording of the supplementary video, and Dr. Kivanc Gungor for fruitful discussions. O.E. acknowledges TUBITAK for the financial support through BIDEB 2211 program. H.V.D. gratefully acknowledges support from TÜBA.

\section{REFERENCES}

(1) Klimov, V. I.; Mikhailovsky, A. A.; Xu, S.; Malko, A.; Hollingsworth, J. A.; Leatherdale, C. A.; Eisler, H. J.; Bawendi, M. G. Optical Gain and Stimulated Emission in Nanocrystal Quantum Dots. Science 2000, 290, 314-317.

(2) Htoon, H.; Hollingworth, J. A.; Malko, A. V.; Dickerson, R.; Klimov, V. I. Light Amplification in Semiconductor Nanocrystals: Quantum Rods versus Quantum Dots. Appl. Phys. Lett. 2003, 82, 4776-4778.

(3) Dang, C.; Lee, J.; Breen, C.; Steckel, J. S.; Coe-Sullivan, S.; Nurmikko, A. Red, Green and Blue Lasing Enabled by Single-Exciton Gain in Colloidal Quantum Dot Films. Nat. Nanotechnol. 2012, 7, 335339.

(4) She, C.; Fedin, I.; Dolzhnikov, D. S.; Demortière, A.; Schaller, R. D.; Pelton, M.; Talapin, D. V. Low-Threshold Stimulated Emission Using Colloidal Quantum Wells. Nano Lett. 2014, 14, 2772-2777.

(5) Guzelturk, B.; Kelestemur, Y.; Gungor, K.; Yeltik, A.; Akgul, M. Z.; Wang, Y.; Chen, R.; Dang, C.; Sun, H.; Demir, H. V. Stable and LowThreshold Optical Gain in CdSe/CdS Quantum Dots: An All-Colloidal Frequency up-Converted Laser. Adv. Mater. 2015, 27, 2741-2746.

(6) Ithurria, S.; Tessier, M. D.; Mahler, B.; Lobo, R. P. S. M.; Dubertret, B.; Efros, A. L. Colloidal Nanoplatelets with TwoDimensional Electronic Structure. Nat. Mater. 2011, 10, 936-941.

(7) Tessier, M. D.; Javaux, C.; Maksimovic, I.; Loriette, V.; Dubertret, B. Spectroscopy of Single CdSe Nanoplatelets. ACS Nano 2012, 6, 6751-6758.

(8) Diroll, B. T.; Talapin, D. V.; Schaller, R. D. Violet-to-Blue Gain and Lasing from Colloidal CdS Nanoplatelets: Low-Threshold Stimulated Emission Despite Low Photoluminescence Quantum Yield. ACS Photonics 2017, 4, 576-583. 
(9) Altintas, Y.; Gungor, K.; Gao, Y.; Sak, M.; Quliyeva, U.; Bappi, G.; Mutlugun, E.; Sargent, E. H.; Demir, H. V. Giant Alloyed Hot Injection Shells Enable Ultralow Optical Gain Threshold in Colloidal Quantum Wells. ACS Nano 2019, 13, 10662-10670.

(10) Guzelturk, B.; Pelton, M.; Olutas, M.; Demir, H. V. Giant Modal Gain Coefficients in Colloidal II-VI Nanoplatelets. Nano Lett. 2019, 19, 277-282.

(11) Guzelturk, B.; Kelestemur, Y.; Olutas, M.; Delikanli, S.; Demir, H. V. Amplified Spontaneous Emission and Lasing in Colloidal Nanoplatelets. ACS Nano 2014, 8, 6599-6605.

(12) She, C.; Fedin, I.; Dolzhnikov, D. S.; Dahlberg, P. D.; Engel, G. S.; Schaller, R. D.; Talapin, D. V. Red, Yellow, Green, and Blue Amplified Spontaneous Emission and Lasing Using Colloidal CdSe Nanoplatelets. ACS Nano 2015, 9, 9475-9485.

(13) Li, Q.; Liu, Q.; Schaller, R. D.; Lian, T. Reducing the Optical Gain Threshold in Two-Dimensional CdSe Nanoplatelets by the Giant Oscillator Strength Transition Effect. J. Phys. Chem. Lett. 2019, 10, 1624-1632.

(14) Sak, M.; Taghipour, N.; Delikanli, S.; Shendre, S.; Tanriover, I.; Foroutan, S.; Gao, Y.; Yu, J.; Yanyan, Z.; Yoo, S.; et al. Coreless FiberBased Whispering-Gallery-Mode Assisted Lasing from Colloidal Quantum Well Solids. Adv. Funct. Mater. 2020, 30, 1907417.

(15) Diroll, B. T.; Greybush, N. J.; Kagan, C. R.; Murray, C. B. Smectic Nanorod Superlattices Assembled on Liquid Subphases: Structure, Orientation, Defects, and Optical Polarization. Chem. Mater. 2015, 27, 2998-3008.

(16) Feng, F.; Nguyen, L. T.; Nasilowski, M.; Nadal, B.; Dubertret, B.; Maitre, A.; Coolen, L. Probing the Fluorescence Dipoles of Single Cubic CdSe/CdS Nanoplatelets with Vertical or Horizontal Orientations. ACS Photonics 2018, 5, 1994-1999.

(17) Paik, T.; Ko, D. K.; Gordon, T. R.; Doan-Nguyen, V.; Murray, C. B. Studies of Liquid Crystalline Self-Assembly of GdF3 Nanoplates by in-Plane, out-of-Plane SAXS. ACS Nano 2011, 5, 8322-8330.

(18) Gao, Y.; Weidman, M. C.; Tisdale, W. A. CdSe Nanoplatelet Films with Controlled Orientation of Their Transition Dipole Moment. Nano Lett. 2017, 17, 3837-3843.

(19) Erdem, O.; Gungor, K.; Guzelturk, B.; Tanriover, I.; Sak, M.; Olutas, M.; Dede, D.; Kelestemur, Y.; Demir, H. V. OrientationControlled Nonradiative Energy Transfer to Colloidal Nanoplatelets: Engineering Dipole Orientation Factor. Nano Lett. 2019, 19, 42974305.

(20) Momper, R.; Zhang, H.; Chen, S.; Halim, H.; Johannes, E.; Yordanov, S.; Braga, D.; Blülle, B.; Doblas, D.; Kraus, T.; et al. Kinetic Control over Self-Assembly of Semiconductor Nanoplatelets. Nano Lett. 2020, 20, 4102-4110.

(21) Chan, Y.; Caruge, J. M.; Snee, P. T.; Bawendi, M. G. Multiexcitonic Two-State Lasing in a CdSe Nanocrystal Laser. Appl. Phys. Lett. 2004, 85, 2460-2462.

(22) Roh, J.; Park, Y. S.; Lim, J.; Klimov, V. I. Optically Pumped Colloidal-Quantum-Dot Lasing in LED-like Devices with an Integrated Optical Cavity. Nat. Commun. 2020, 11, 271.

(23) Liu, B.; Altintas, Y.; Wang, L.; Shendre, S.; Sharma, M.; Sun, H.; Mutlugun, E.; Demir, H. V. Record High External Quantum Efficiency of $19.2 \%$ Achieved in Light-Emitting Diodes of Colloidal Quantum Wells Enabled by Hot-Injection Shell Growth. Adv. Mater. 2020, 32, 1905824.

(24) Beck, M.; Graczyk, M.; Maximov, I.; Sarwe, E. L.; Ling, T. G. I.; Keil, M.; Montelius, L. Improving Stamps for $10 \mathrm{Nm}$ Level Wafer Scale Nanoimprint Lithography. Microelectron. Eng. 2002, 61-62, 441-448.

(25) Min, H.; Zhou, J.; Bai, X.; Li, L.; Zhang, K.; Wang, T.; Zhang, X.; Li, Y.; Jiao, Y.; Qi, X.; et al. Approach to Fabricating a Compact Gold Nanoparticle Film with the Assistance of a Surfactant. Langmuir 2017, $33,6732-6738$.

(26) Decher, G. Fuzzy Nanoassemblies: Toward Layered Polymeric Multicomposites. Science 1997, 277, 1232-1237.

(27) Roither, J.; Pichler, S.; Kovalenko, M. V.; Heiss, W.; Feychuk, P.; Panchuk, O.; Allam, J.; Murdin, B. N. Two- And One-Dimensional Light Propagations and Gain in Layer-by-Layer-Deposited Colloidal Nanocrystal Waveguides. Appl. Phys. Lett. 2006, 89, 111120.
(28) Ozel, T.; Hernandez-Martinez, P. L.; Mutlugun, E.; Akin, O.; Nizamoglu, S.; Ozel, I. O.; Zhang, Q.; Xiong, Q.; Demir, H. V. Observation of Selective Plasmon-Exciton Coupling in Nonradiative Energy Transfer: Donor-Selective versus Acceptor-Selective Plexcitons. Nano Lett. 2013, 13, 3065-3072.

(29) Suarez, I.; Munoz, R.; Chirvony, V.; Martinez-Pastor, J. P.; Artemyev, M.; Prudnikau, A.; Antanovich, A.; Mikhailov, A. Multilayers of CdSe/CdS/ZnCdS Core/Wings/Shell Nanoplatelets Integrated in a Polymer Waveguide. IEEE J. Sel. Top. Quantum Electron. 2017, 23, 1-8.

(30) Halthur, T. J.; Claesson, P. M.; Elofsson, U. M. Stability of Polypeptide Multilayers as Studied by in Situ Ellipsometry: Effects of Drying and Post-Buildup Changes in Temperature and PH. J. Am. Chem. Soc. 2004, 126, 17009-17015.

(31) Hammond, P. T. Engineering Materials Layer-by-Layer: Challenges and Opportunities in Multilayer Assembly. AIChE J. 2011, 57, 2928-2940.

(32) Dong, A.; Chen, J.; Vora, P. M.; Kikkawa, J. M.; Murray, C. B. Binary Nanocrystal Superlattice Membranes Self-Assembled at the Liquid-Air Interface. Nature 2010, 466, 474-477.

(33) Lambert, K.; Čapek, R. K.; Bodnarchuk, M. I.; Kovalenko, M. V.; Van Thourhout, D.; Heiss, W.; Hens, Z. Langmuir-Schaefer Deposition of Quantum Dot Multilayers. Langmuir 2010, 26, 7732-7736.

(34) Vogel, N.; De Viguerie, L.; Jonas, U.; Weiss, C. K.; Landfester, K. Wafer-Scale Fabrication of Ordered Binary Colloidal Monolayers with Adjustable Stoichiometries. Adv. Funct. Mater. 2011, 21, 3064-3073.

(35) Shaklee, K. L.; Nahory, R. E.; Leheny, R. F. Optical Gain in Semiconductors. J. Lumin. 1973, 7, 284-309.

(36) Altintas, Y.; Quliyeva, U.; Gungor, K.; Erdem, O.; Kelestemur, Y.; Mutlugun, E.; Kovalenko, M. V.; Demir, H. V. Highly Stable, NearUnity Efficiency Atomically Flat Semiconductor Nanocrystals of CdSe/ $\mathrm{ZnS}$ Hetero-Nanoplatelets Enabled by ZnS-Shell Hot-Injection Growth. Small 2019, 15, 1804854.

(37) Rossinelli, A. A.; Rojo, H.; Mule, A. S.; Aellen, M.; Cocina, A.; De Leo, E.; Schäublin, R.; Norris, D. J. Compositional Grading for Efficient and Narrowband Emission in CdSe-Based Core/Shell Nanoplatelets. Chem. Mater. 2019, 31, 9567-9578.

(38) Zhang, L.; Yang, H.; Yu, B.; Tang, Y.; Zhang, C.; Wang, X.; Xiao, M.; Cui, Y.; Zhang, J. Low-Threshold Amplified Spontaneous Emission and Lasing from Thick-Shell CdSe/CdS Core/Shell Nanoplatelets Enabled by High-Temperature Growth. Adv. Opt. Mater. 2020, 8, 1901615.

(39) Sheridan, A. K.; Turnbull, G. A.; Safonov, A. N.; Samuel, I. D. W. Tuneability of Amplified Spontaneous Emission through Control of the Waveguide-Mode Structure in Conjugated Polymer Films. Phys. Rev. B: Condens. Matter Mater. Phys. 2000, 62, R11929.

(40) Calzado, E. M.; Villalvilla, J. M.; Boj, P. G.; Quintana, J. A.; DíazGarcía, M. A. Tuneability of Amplified Spontaneous Emission through Control of the Thickness in Organic-Based Waveguides. J. Appl. Phys. 2005, 97, No. 093103.

(41) Yariv, A.; Yeh, P. Photonics: Optical Electronics in Modern Communications; Oxford University Press: New York, NY, 2007.

(42) Klimov, V. I.; Ivanov, S. A.; Nanda, J.; Achermann, M.; Bezel, I.; McGuire, J. A.; Piryatinski, A. Single-Exciton Optical Gain in Semiconductor Nanocrystals. Nature 2007, 447, 441-446. 